\title{
Assessment of a BeagleBone Black High Sampling Rate Digital Waveform Generator
}

\author{
Kevin Verniers, Liesbet Van der Perre, Nobby Stevens \\ KU Leuven, ESAT-DRAMCO, Ghent Technology Campus \\ Ghent, Belgium \\ kevin.verniers@kuleuven.be
}

\begin{abstract}
Modulation waveform generators are frequently deployed for numerous applications such as communications. As a low-cost solution for high sampling rates, we investigate the BeagleBone Black (BBB) and more specific its Programmable Real-Time Unit (PRU) subsystem, which supports time critical tasks and fast deterministic IO operations. The implementation overview along with the achieved results in terms of throughput and reliability are herein presented and explained. The feasibility of a BBB PRU-based digital waveform generator was demonstrated under varying output configurations (up to 13) and sampling rates (up to 50 MSPS) with a high reliability ( $>$ 95\%).
\end{abstract}

\section{INTRODUCTION}

Many prototype realizations such as a Visible Light Communications (VLC) prototype require a waveform generator to modulate a signal, in this case the emitted light intensity by adjusting the LED current. Depending on the modulation properties, an analog or digital waveform representation is preferred. In this work, the focus is on the digital variant, concerning the VLC case some possibilities are On-Off Keying (OOK), Variable Pulse Position Modulation (VPPM), and Single Edge Position Modulation (SEPM). The first two are defined in the IEEE 802.15.7 VLC standard [1], whereas the latter is proposed by Stevens's work in [2]. Low frequency waveform reconstruction requires a low sampling rate, which is typically in the order of several thousands of Samples Per Second (kSPS). For this purpose, many commercial low-cost waveform generators are already available. If a high frequency waveform is required, the sampling rate rapidly increases in the order of tens of MSPS. Several manufacturers provide commercially available arbitrary waveform generators fulfilling these high sampling rates e.g., National Instruments - PXI series ${ }^{1}$, Tektronix - AWG series ${ }^{2}$. However, these are expensive and do not necessarily fit the low-cost requirement for prototype realization.

Wang et Al. [3] proposed an open source research platform for VLC, openVLC, based on the BeagleBone Black (BBB), a low-cost Single-Board Computer (SBC). In the frame of their experimental evaluation, a performance enhancement

\footnotetext{
${ }^{1}$ https://www.ni.com/nl-be/shop/select/pxi-waveform-generator

${ }^{2}$ https://www.tek.com/arbitrary-waveform-generator/awg5200
}

978-1-5386-7557-1/18/\$31.00 (C)2018 European Union list was proposed, where they suggest the deployment of the embedded Programmable Real-Time Unit (PRU) subsystem in the BBB's processor. Fedrizzi and Soria utilized this PRU subsystem, as elaborated in [4], in order to create a pulse generator where the desired square pulse is preloaded once into the PRU subsystem and afterwards repetitively exported towards an output pin. Furthermore, they provided a performance comparison of their pulse generator with commercial available devices in terms of RMS jitter and timing base stability.

A similar PRU project was realized by Tarratt [5] to perform a Direct Digital Synthesizer (DDS) with a sampling rate of 28.5 MSPS. The desired waveform is preloaded as a table into the PRU subsystem $8 \mathrm{kB}$ RAM. However, in case of modulation waveforms exceeding $8 \mathrm{kB}$, a larger memory capacity is needed.

Abhishek's work [6] resulted into a high sampling rate (100 MSPS), high memory capacity (up to $320 \mathrm{MB}$ ) Logic Analyzer (LA) based on the PRU subsystem. In this paper, the LA framework from [6] was adapted to create and assess a digital waveform generator capable of producing one or several long streams of OOK, VPPM, SEPM, or another digital waveform at a high sampling rate. The remainder of this paper is organized as follows: Section II describes the high level implementation overview, whereas Section III provides a more detailed explanation concerning the PRU subsystem and the possible output configurations. Section IV contains the waveform generator benchmark for each output configuration. Section V summarizes the main findings of this realization and assessment.

\section{SySTEM OVERVIEW}

A Modern BBB is equipped with the Octavo Systems OSD3358 System-in-Packages (SiP) device, which integrates several elements such as the AM3358 Sitara processor from Texas Instruments (TI), the DDR3 RAM, and a Power Management Integrated Circuit (PMIC). The main focus is on the AM3358 processor, which contains the PRU subsystem. The LA framework provided by Abhishek in [6] is reused, however, several communication directions are reversed because of the goal to generate digital waveforms instead of capturing them. The adapted framework is given in Fig. 1 where each of the 
four deployed components is briefly discussed in a separate subsection.

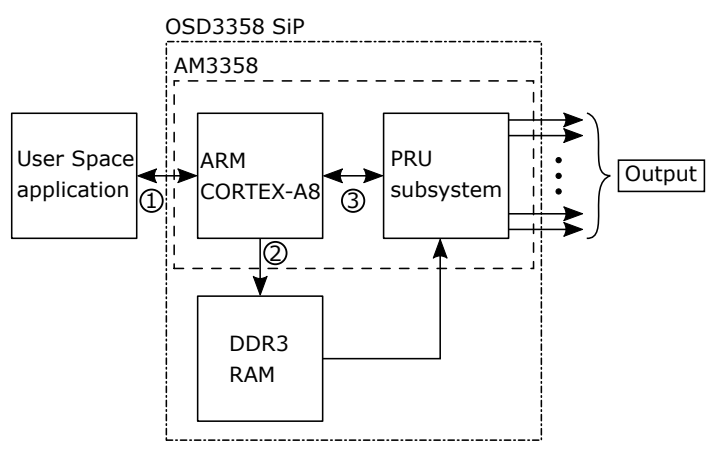

Fig. 1. Simplified overview the digital waveform generator's main components and communication directions, adapted from [6].

\section{A. PRU Subsystem}

The waveform generator's heart is the PRU subsystem, which is embedded in the AM3358 processor to handle critical real-time tasks by assuring a fast deterministic operation. The list below briefly summarizes some available features. An elaborated list can be found in the processor technical reference manual ${ }^{3}$.

\section{- 2 PRU cores (PRU0 and PRU1)}

- 32-bit processor

- $200 \mathrm{MHz}$ clock frequency

- 32 32-bit registers

- Scratch Pad memory (SPAD)

- 3 banks of 30 32-bit registers

- Interrupt Controller (INTC)

- 64 interrupt events

The PRU subsytem's role in the waveform generator is extensively discussed in Section III.

\section{B. ARM Cortex-A8}

The ARM cortex-A8 is integrated in the AM3358 processor and operates at a $1 \mathrm{GHz}$ clock frequency to provide a high processing capability. Furthermore, a Linux Operating System such as Debian or Ubuntu can be deployed.

The ARM communicates with three components: (1) a User Space application, (2) the DDR3 RAM, and (3) the PRU subsystem. The first link (indicated (1) in Fig. 1) exchanges the complete desired waveform(s) from the User Space as well as commands such as "start" and "finish" to or from the User Space. The second link (marked (2) in Fig. 1) stores the desired waveform(s) in the DDR3 RAM. The storage realization is determined by the ARM and explained in Section II-C. The last link (3) in Fig. 1) exchanges address information about the storage realization inside the RAM as well as the commands to or from the PRU subsystem.

\footnotetext{
${ }^{3}$ https://www.ti.com/lit/ug/spruh73p/spruh73p.pdf
}

\section{DDR3 RAM Memory}

The OSD3358 SiP is equipped with a 512 MB DDR3 RAM to store the waveform(s). The storage structure is based on [6], which utilized circularly linked, uniform size buffers. Here, a similar structure is deployed except for the last buffer's size, which depends on the remaining samples to output in order to prevent the generation of undesired samples.

\section{User Space Application}

A programming language such as Python or $\mathrm{C}$ can be used to both create and exchange the desired waveform(s) to the ARM, and to start the waveform generator. Because of different output configurations, it is important to correctly multiplex the samples of each waveform, which is explained in Section III-C.

\section{WAVEForm Generation CORE: THE PRU SUBSYSTEM}

In Abhishek's work in [6], each PRU core fulfills a dedicated functionality along with the SPAD which provides flexibility. In this work, the PRU cores functionalities are maintained except that they are now configured to fulfill a waveform generator operation as indicated in Fig. 2. The following subsections provide a detailed discussion about these three components.

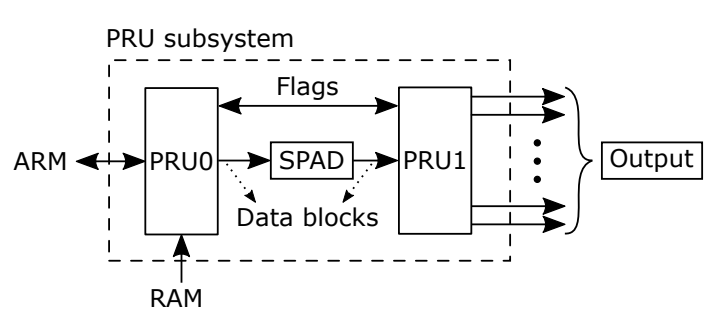

Fig. 2. Overview of the PRU subsystem internal and external connections, adapted from [6].

\section{A. SPAD: Flexibility}

The SPAD is deployed to transfer data from or to a PRU core, which enables an indirect data block transfer between PRU0 and PRU1. A striking feature is the single clock cycle execution which transfers up to 30 registers, or 120 bytes, between a PRU core and a bank, out of three, of the SPAD. In [6], the data block size was limited to 32 bytes, we extended this to 64 bytes in order to investigate the impact on the modulation waveform generator in terms of reliability at an increasing sampling rate and/or number of outputs (Section IV). Here, reliability is defined as the correct data block transfer in time between PRU0 and PRU1.

\section{B. PRUO: Data Management}

PRU0 deals with the data management part where it is configured to retrieve the data blocks from the RAM and to process them towards the SPAD. Furthermore, PRU0 communicates with both the ARM and PRU1 to ensure a 
correct data management, which is divided into two stages.

During the first stage, before starting the waveform generator, address information concerning the storage structure inside the RAM is exchanged between PRU0 and the ARM, which enables Direct Memory Access (DMA) by PRU0 once the waveform generator starts. The User Space application sends the "start" command to the ARM, which in turn notifies PRU0 in order to initiate the waveform generator.

In the second stage, PRU0 transfers the first data block, 32 or 64 bytes, from the RAM into the SPAD. Then, PRU1 is triggered by PRU0, via Flags in Fig. 2, in order to process this first data block towards the output pins. Meanwhile, PRU0 waits until PRU1 requests, also via Flags in Fig. 2, to load the next data block into the SPAD. This operation continues until the complete modulation waveform is generated at the output allowing PRU0 to send the "finished" command to the ARM, which in turn notifies the User Space application that the whole process has ended.

An important challenge to achieve a high reliability, is the duration to load the data blocks from the RAM into PRUO. This operation has a non-deterministic behavior because the RAM is an external component with respect to the PRU subsystem (see Fig. 1). Thus, PRU0 must be able to both load the next data block from the RAM and to store it into the SPAD before PRU1 copies this data from the SPAD. In Section IV-A2, a measurement is presented that provides insight in this non-deterministic behavior for both 32 and 64 bytes data block size.

\section{PRU1: Output Configuration and Sampling Rate}

In previous work [6], PRU1 retrieves data from the input pins and transfers it onto the SPAD. Afterwards, PRU1 triggers PRU0 that a valid data block is available at the SPAD. In this work, PRU1's operation is reversed and now it requests, via Flags in Fig. 2, PRU0 to fill the SPAD with a valid data block. Thus, after PRU1 is triggered by PRU0 at the startup of the waveform generator, it copies the first data block from the SPAD and processes this towards the output pins at the desired sampling rate. In the meantime, PRU1 requests PRU0 to load the next data block into the SPAD.

Since no connections outside the PRU subsystem are necessary, PRU1's operation has a fixed duration, and thus a deterministic behavior. This results in a fixed timing constraint for PRU0 to both load from the RAM and to store the next data block into the SPAD. This timing constraint depends on two elements: (1) the data block size, and (2) the number of deployed output pins. Here, three possible output configurations for the number of output pins were considered: 4,8 , or 13 (maximum). The last value is limited due to the number of physically accessible PRU1 output pins on the BBB's pin headers as listed by Molloy in [7].
Section II-D stated the importance to correctly multiplex the modulation waveforms regarding the applied output configuration. Fig. 3 shows the multiplexing step for one register of PRU1 for each considered output configuration.

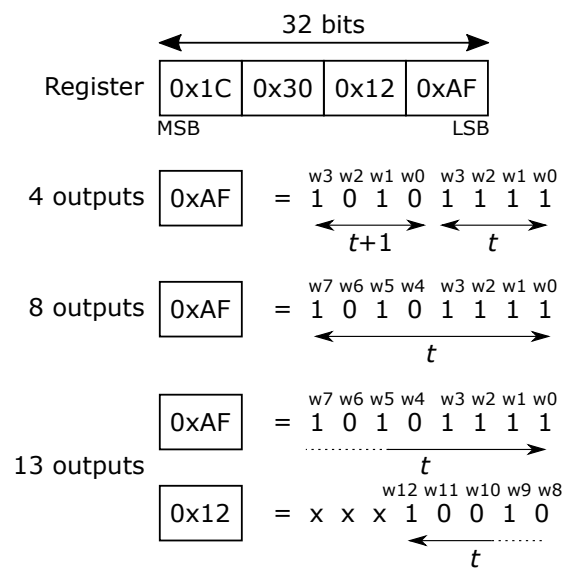

w0 = waveform $0 ;$ w1 $=$ waveform $1 ; \ldots$ $t=$ sampling time instant

Fig. 3. Multiplexing step for one register of the configurations: 4 , 8 , or 13 outputs. In order to store a sample of each waveform, the case of 4 outputs requires a nibble; 8 outputs requires a byte; 13 outputs requires 2 bytes.

Each register carries 32 bits. Depending on the number of deployed output pins, which equals the number of possible modulation waveforms, a register carries a certain amount of samples of each modulation waveform. If 4 outputs are deployed, a nibble is sufficient to carry a sample of each waveform resulting in a total of 8 samples of each waveform for one register. For 8 outputs, a byte is necessary to hold a sample of each waveform resulting in only 4 samples of each waveform to be stored in one register. If 13 outputs are desired, it takes two bytes to store a sample of each waveform resulting in two samples of each waveform to be stored in one register.

The data block processing is executed by propagating the adjacent samples of each waveform towards the output pins at the desired sampling rate, which is set by PRU1 which operates at $200 \mathrm{MHz}$. Therefore, PRU1 should wait one or several clock cycles before propagating the next sample towards the output pins. Consider Fig. 4 when 8 outputs and a 100 MSPS are required. Furthermore, keep in mind the register values from Fig. 3.

$$
\begin{aligned}
& \begin{array}{ll|l|l|l|l|l|}
\hline \text { PRU1 } & \text { S1 } & \mathrm{N} & \mathrm{S} 2 & \mathrm{~S} 3 & \mathrm{~S} 4 \\
\hline
\end{array}
\end{aligned}
$$

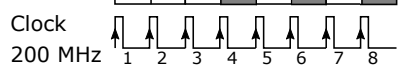

$$
\begin{aligned}
& \mathrm{Sx}=\mathrm{X}^{\text {th }} \text { sample of each waveform } \\
& \mathrm{N}=\text { notify PRU0 for next data block } \\
& =\text { PRU1 waits }
\end{aligned}
$$

Fig. 4. Propagating samples $\mathrm{Sx}$ towards the output pins when a $100 \mathrm{MHz}$ sampling rate is set. PRU1's clock frequency is $200 \mathrm{MHz}$, therefore it has to wait one clock cycle before propagating the next sample of each waveform. 
At the first clock cycle, the first sample S1 from each waveform is propagated towards the output pins, in this case the value "OxAF". The next clock cycle, PRU1 notifies (N) PRU0 to load the next data block into the SPAD. The third clock cycle, the next sample S2 of each waveform is propagated towards the output pins, here "0x12". The fourth clock cycle, PRU1 just waits. The fifth clock cycle, PRU1 propagates S3 towards the outputs, now the value " $0 \times 30$ ". At the sixth clock cycle PRU1 waits again. This process repeats until a complete data block is propagated towards the output pins. Afterwards, PRU1 copies the new data block from the SPAD and the data block processing starts again from the beginning. This continues until PRU0 stops the waveform generator, i.e. when all data blocks are processed. The example clarifies the maximum achievable sampling rate of 100 MSPS because of the necessary communication between PRU0 and PRU1. However, due to the external RAM latencies the maximum achievable sampling rate which achieves a high reliability (over $95 \%$ ) will decrease. If a lower sampling rate is desired, PRU1 simply waits several clock cycles before propagating the next sample of each waveform.

\section{BENCHMARK}

To assess the waveform generator, two measurement types were conducted. First, timing information was collected from the following two elements in Fig. 2.

- PRU1: Data Block Processing

- PRU0: Data Management and Transfer

Second, the waveform generator output was monitored to verify the data block transfer correctness from the RAM via PRU0 to PRU1 for the considered output configurations.

\section{A. Timing Information}

Both PRU cores contain a clock cycle counter that increments on each rising edge of the $200 \mathrm{MHz}$ clock. Therefore, the following results are displayed in terms of necessary clock cycles.

1) PRU1: Data Block Processing: As mentioned in Section III-C, PRU1 has a complete deterministic operation, whose duration, which forms the timing constraint for PRU0, depends on two elements: (1) the output configuration, and (2) the data block size. Table I shows the necessary clock cycles to process a data block for each considered output configuration. Logically, this increases when: (1) the data block size increases, (2) the targeted sample rate decreases, or (3) the number of output pins decreases.

2) PRU0: Data Management and Transfer: PRU0's operation contains a deterministic and non-deterministic part. The first part results from data management tasks such as inspecting the current buffer's data, accessing the next buffer, storing data into the SPAD, and finishing the waveform generator operation. The latter part results from data transfers from the RAM to PRU0. The complete operation must be executed within the timing constraints dictated by PRU1,
TABLE I

PRU1 Data Block Processing: Necessary Clock Cycles

\begin{tabular}{|c|c|c|c|c|c|c|}
\hline \multirow{2}{*}{$\begin{array}{c}\text { Sampling Rates } \\
\text { (MSPS) }\end{array}$} & \multicolumn{5}{|c|}{ D2 Bytes } & \multicolumn{3}{c|}{ 64 Bytes } \\
\cline { 2 - 7 } & \multicolumn{3}{|c|}{$\mathbf{N}^{\circ}$ Outputs } & \multicolumn{3}{c|}{ Outputs } \\
\cline { 2 - 7 } & $\mathbf{4}$ & $\mathbf{8}$ & $\mathbf{1 3}$ & $\mathbf{4}$ & $\mathbf{8}$ & $\mathbf{1 3}$ \\
\hline $\mathbf{2 5}$ & 512 & 256 & 128 & 1024 & 512 & 256 \\
\hline $\mathbf{3 3 . 3 3}$ & 384 & 192 & 96 & 768 & 384 & 192 \\
\hline $\mathbf{5 0}$ & 256 & 128 & 64 & 512 & 256 & 128 \\
\hline
\end{tabular}

which are listed in Table I.

Fig. 5 shows a cropped time series of necessary clock cycles for both deterministic and non-deterministic part when a 32 byte data block size is chosen.

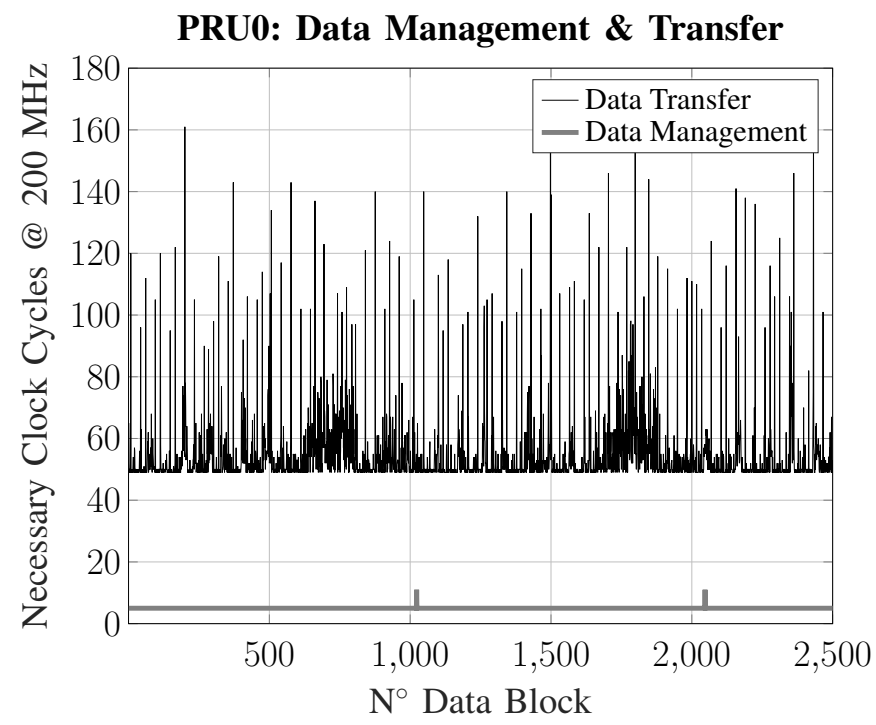

Fig. 5. PRU0 data management and transfer timing information in case of a 32 byte data block size. Accessing the next buffer results into small peaks in the Data Management result.

The latter part requires significantly more clock cycles than the first part, which will reduce the waveform generator's reliability when a higher sampling rate and/or more output pins are required. If the data block size is augmented to 64 bytes, the deterministic part remains unchanged while this is not valid for the non-deterministic part. Further insight is provided by two scatter plots, which actually represent two histograms, in Figs. 6 and 7. These show the necessary clock cycle distribution and some statistical parameters over one million data block transfers for both 32 and 64 bytes data block size, respectively.

We conclude from both scatter plots that a doubled data block size does not result, in this case, into a doubling of necessary clock cycles. However, it introduces a higher minimum, a higher maximum, and a larger variance of the clock cycle distribution. Despite the latter, the mean and median do not deviate significantly from the minimum. Presumably, occasionally active background processes might interrupt the data 
block transfer from the RAM memory to PRU0 by occupying the data transfer bus leading to a larger variance.

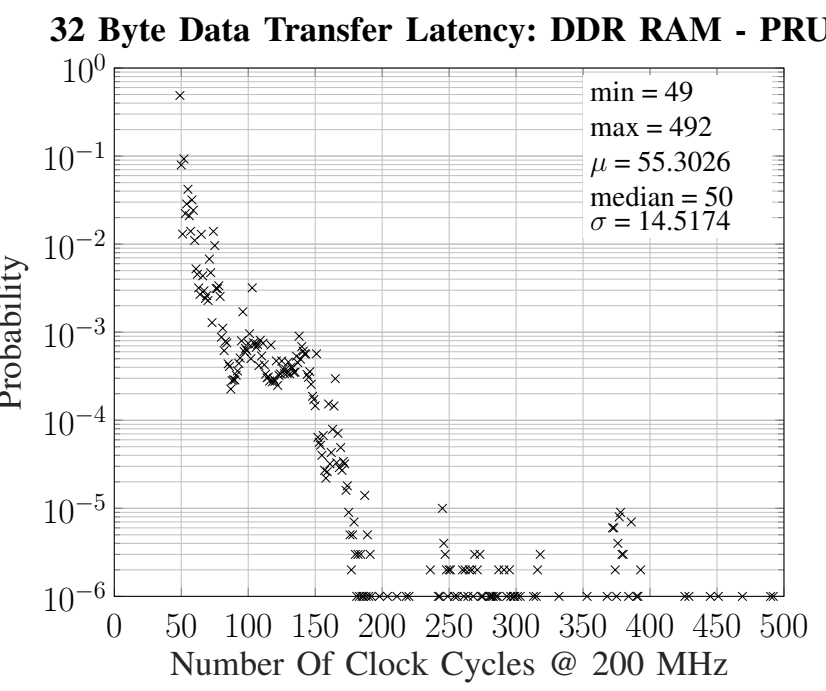

Fig. 6. Scatter plot of the 32 byte data block transfer latency from the RAM memory to PRU0.

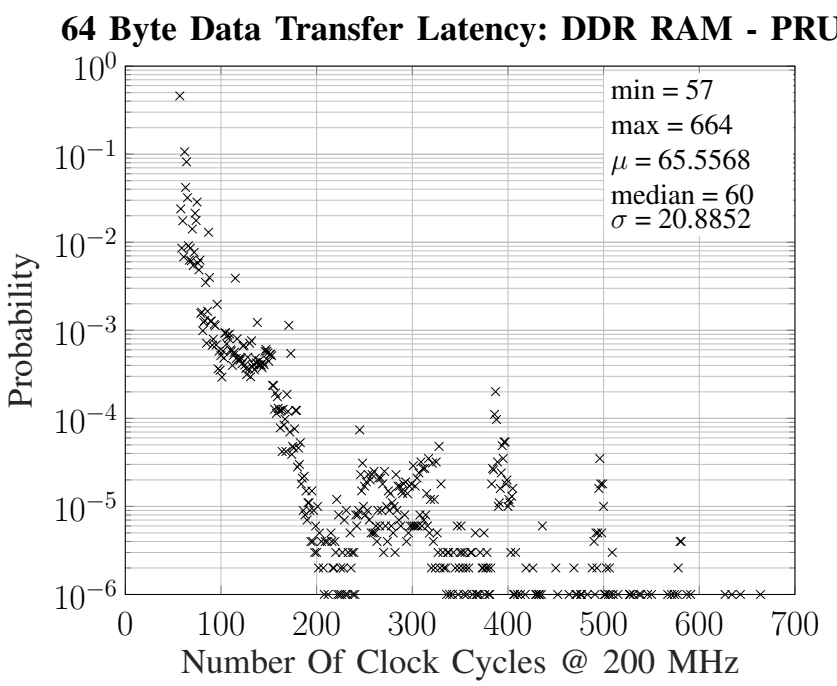

Fig. 7. Scatter plot of the 64 byte data block transfer latency from the RAM memory to PRU0.

To calculate the waveform generator's reliability, the information from Table I is combined with both scatter plots and the reliability results are given in Table II. Despite a larger variance, a 64 byte data block size achieves a higher reliability over the considered output configurations in comparison to a 32 byte data block size. This results from: (1) a limited increase of additional clock cycles, 8 when both minimum values are compared, to load 64 bytes from the RAM to PRU0, and (2) a less strict timing constraint set by PRU1, because a larger data block size results in an extended data block processing time.

Still, further improvements are necessary to decrease this variance in order to achieve a $100 \%$ reliability over all pos-
TABLE II

CONFIgurations' Reliability $(\%)$

\begin{tabular}{|c|c|c|c|}
\hline \multirow{2}{*}{$\begin{array}{c}\text { Sampling Rate } \\
\text { (MSPS) }\end{array}$} & \multicolumn{3}{|c|}{ Data Block Size: 32 Bytes } \\
\cline { 2 - 4 } & $\mathbf{3}$ & $\mathbf{N}^{\circ}$ Outputs \\
\cline { 2 - 4 } & $\mathbf{4}$ & $\mathbf{8}$ & $\mathbf{1 3}$ \\
\hline $\mathbf{2 5}$ & 100 & 99.9864 & 98.4101 \\
\hline $\mathbf{3 3 . 3 3}$ & 99.9945 & 99.9820 & 96.3693 \\
\hline $\mathbf{5 0}$ & 99.9864 & 98.4101 & 67.2864 \\
\hline
\end{tabular}

\begin{tabular}{|c|c|c|c|}
\hline \multirow{2}{*}{$\begin{array}{c}\text { Sampling Rates } \\
\text { (MSPS) }\end{array}$} & \multicolumn{3}{|c|}{ Data Block Size: 64 Bytes } \\
\cline { 2 - 4 } $\mathbf{N}^{\circ}$ outputs \\
\cline { 2 - 4 } $\mathbf{2 5}$ & $\mathbf{4}$ & $\mathbf{8}$ & $\mathbf{1 3}$ \\
\hline $\mathbf{3 3 . 3 3}$ & 100 & 99.9960 & 99.7633 \\
\hline $\mathbf{5 0}$ & 99.9960 & 99.8916 & 99.7179 \\
\hline & &
\end{tabular}

sible configurations. Three possible improvements we suggest are: (1) use of a Real-Time Operating System (RTOS), (2) study of the On-Chip Protocol (OCP), which couples the PRU subsystem with external components such as the RAM, (3) priorization of the DMA transfer over the background processes that also use the data transfer bus. If successful, the outliers will be reduced and will lead to a higher reliability at a higher sampling rate and/or more deployed outputs.

\section{B. Modulation Waveform Generator Output}

One output pin of each considered configuration was captured with an oscilloscope in order to verify the data block transfer correctness from the RAM via PRU0 to PRU1. In Fig. 8, a result is given for 8 outputs, a 32 byte data block size, and a sample rate of $25 \mathrm{MHz}$.

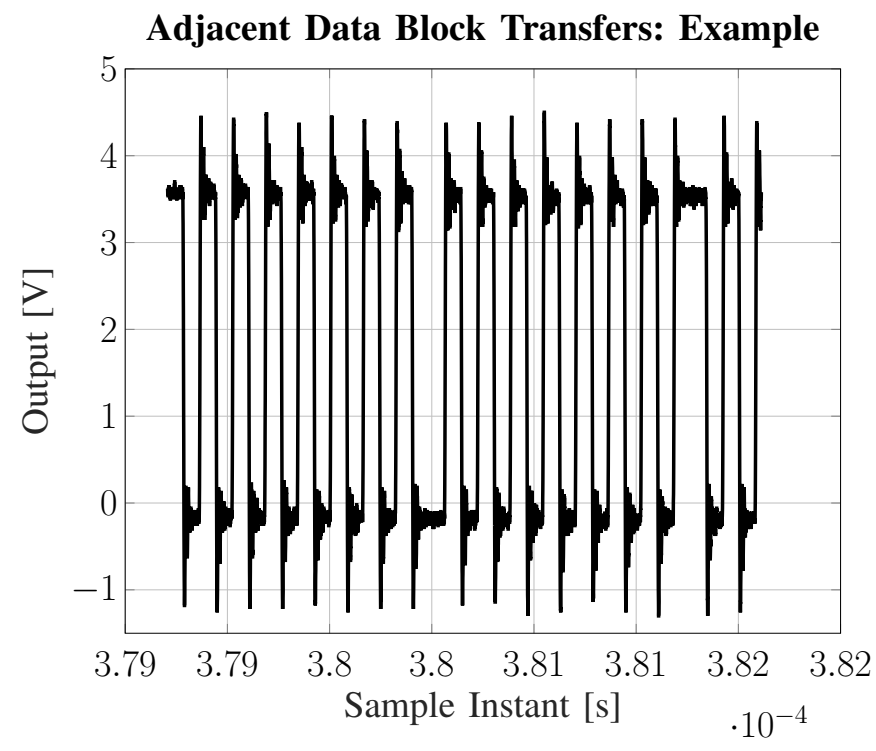

Fig. 8. Adjacent data block transfers for 8 outputs, 32 bytes data block size, and 25 MSPS. Adjacent data blocks contain mirrored square waves, and a correct data block transfer is indicated by an extended edge transition duration.

In the result, adjacent data blocks contain a mirrored waveform, which allows easier data block detection by means of an extended edge transition duration between those data blocks. 
If at specific intervals no extended edge transition duration is detected, then the old data block was retransmitted instead of the new one, indicating an erroneous reconstruction at that moment. By means of this method the values listed in Table III for each output configuration were obtained. The total number of data blocks transfers was limited to 2500 because of insufficient oscilloscope memory. From Table III, similar conclusions are drawn as from Table II thus validating the earlier conclusions.

TABLE III

DATA BLOCK TRANSFERS: ERRONEOUS TRANSFERS AT 2500 DATA BLOCK TRANSFERS

\begin{tabular}{|c|c|c|c|c|c|c|}
\hline \multirow{2}{*}{$\begin{array}{c}\text { Sampling Rates } \\
\text { (MSPS) }\end{array}$} & \multicolumn{5}{|c|}{ Data Block Size } \\
\cline { 2 - 7 } & \multicolumn{3}{|c|}{ 32 Bytes } & \multicolumn{3}{c|}{ 64 Bytes } \\
\cline { 2 - 7 } & \multicolumn{2}{|c}{ Outputs } & \multicolumn{3}{c|}{$\mathbf{N}^{\circ}$ Outputs } \\
\cline { 2 - 7 } & $\mathbf{4}$ & $\mathbf{8}$ & $\mathbf{1 3}$ & $\mathbf{4}$ & $\mathbf{8}$ & $\mathbf{1 3}$ \\
\hline $\mathbf{2 5}$ & 0 & 2 & 114 & 0 & 0 & 0 \\
\hline $\mathbf{3 3 . 3 3}$ & 0 & 0 & 160 & 0 & 2 & 2 \\
\hline $\mathbf{5 0}$ & 2 & 84 & 716 & 0 & 0 & 124 \\
\hline
\end{tabular}

\section{CONCLUSION}

In this work, the feasibility is demonstrated of a BeagleBone Black (BBB) high sampling rate digital waveform generator with a large memory capacity by deploying the Programmable Real-Time Unit (PRU) subsystem. Earlier PRU projects from [4], [5] generate square waveforms, however, both only consider fixed waveform types, which are stored in the PRU subsystem internal RAM. In [6], the PRU subsystem is deployed to realize a 100 MSPS Logic Analyzer with a $320 \mathrm{MB}$ storage, whereas here the necessary adaptations are made to realize an arbitrary digital waveform generator supporting up to 13 output pins and a sampling rate up to 50 MSPS. The modulation waveform is processed in data blocks towards the output pins, two data block sizes were considered: 32 and 64 bytes. The conducted measurements have shown an increased reliability, almost $100 \%$, at higher sampling rates and/or more output pins for the 64 byte data block size. However, further improvements are still necessary to achieve a $100 \%$ reliability for all output configurations. Therefore, several measures that may benefit the waveform generator were proposed in this work. The waveform generator framework can be found on Github entitled "PRU Digital Waveform Generator""

\section{ACKNOWLEDGMENT}

The authors thank Research Foundation - Flanders (FWO) for the financial support of $\mathrm{SB} \mathrm{PhD}$ fellow at FWO, Kevin Verniers, project number: 1S09617N.

\section{REFERENCES}

[1] "IEEE Standard for Local and Metropolitan Area Networks-Part 15.7: Short-Range Wireless Optical Communication Using Visible Light," in IEEE Std 802.15.7-2011, vol., no., pp.1-309, Sept. 62011

[2] N. Stevens and L. De Strycker, "Single Edge Position Modulation as a Dimming Technique for Visible Light Communications," in Journal of Lightwave Technology, vol. 34, no. 23, pp. 5554-5560, Dec.1, 12016.

${ }^{4}$ https://github.com/VerniersK/PRU_Digital_Waveform_Generator
[3] Q. Wang, D. Giustiniano and D. Puccinelli, "An open source research platform for embedded visible light networking," in IEEE Wireless Communications, vol. 22, no. 2, pp. 94-100, April 2015.

[4] M. Fedrizzi, J. Soria, "Application of a single-board computer as a low cost pulse generator," in Measurement Science and Technology, vol. 26 , no. 9, pp. 095302, April 2015.

[5] B. Tarratt, pru-dds, (2014), GitHub repository, https://github.com/n8vi/pru-dds

[6] K. Abhishek, BeagleLogic, (2014), Github repository, https://github.com/abhishek-kakkar/BeagleLogic/wiki

[7] D. Molloy, Exploring Beaglebone, 1st ed. Indianapolis, John Wiley \& Sons, Inc., pp. 211-212, 2015 